\section{(2) OPEN ACCESS}

\begin{abstract}
- Additional material is published online only. To view, please visit the journal online (http://dx.doi.org/10.1136/ heartjnl-2020-316920).
\end{abstract}

For numbered affiliations see end of article.

Correspondence to Dr Ben Hurdus, Leeds Institute for Data Analytics, Leeds Institute of Cardiovascular and Metabolic Medicine, Leeds LS2 9LU, UK; ben.hurdus@nhs.net

This work was due to be presented as a moderated poster presentation at the Acute Cardiovascular Care Congress in Athens 7-9 of March 2020; however, due to the COVID-19 pandemic, the congress was cancelled. The abstract and poster will be published in a special edition abstract supplement to the European Heart Journal, Acute Cardiovascular Care Association for Acute CardioVascular Care.

Received 20 March 2020 Revised 30 June 2020

Accepted 14 July 2020 Published Online First 21 August 2020

\section{Linked}

- http://dx.doi.org/10.1136/ heartjnl-2020-317497

\section{Check for updates}

(c) Author(s) (or their employer(s)) 2020. Re-use permitted under CC BY. Published by BMJ.

To cite: Hurdus $B$

Munyombwe $T$,

Dondo TB, et al. Heart

2020;106:1726-1731.

\title{
Association of cardiac rehabilitation and health- related quality of life following acute myocardial infarction
}

\author{
Ben Hurdus (D) ,',2 Theresa Munyombwe (D) ,' Tatendashe Bernadette Dondo (D) , \\ Suleman Aktaa, ${ }^{1,2}$ Gerrard Oliver, ${ }^{3}$ Marlous Hall, ${ }^{1}$ Patrick Doherty, ${ }^{4}$ Alistair S Hall, ${ }_{1}^{1,2}$ \\ Chris P Gale ${ }^{1,2}$
}

\section{ABSTRACT \\ Objective To study the association of cardiac} rehabilitation and physical activity with temporal changes in health-related quality of life (HRQoL) following acute myocardial infarction (AMI).

Methods Evaluation of the Methods and Management of Acute Coronary Events-3 is a nationwide longitudinal prospective cohort study of 4570 patients admitted with an AMI between 1 November 2011 and 17 September 2013. HRQoL was estimated using EuroQol 5-Dimension-3 Level Questionnaire at hospitalisation, 30 days, and 6 and 12 months following hospital discharge. The association of cardiac rehabilitation and selfreported physical activity on temporal changes in $\mathrm{HRQ}$ oL was quantified using inverse probability of treatment weighting propensity score and multilevel regression analyses.

Results Cardiac rehabilitation attendees had higher HRQoL scores than non-attendees at 30 days (mean EuroQol 5-Visual Analogue Scale (EQ-VAS) scores: 71.0 (SD 16.8) vs 68.6 (SD 19.8)), 6 months (76.0 (SD 16.4) vs 70.2 (SD 19.0)) and 12 months (76.9 (SD 16.8) vs 70.4 (SD 20.4)). Attendees who were physically active $\geq 150$ min/week had higher HRQoL scores compared with those who only attended cardiac rehabilitation at 30 days (mean EQ-VAS scores: 79.3 (SD 14.6) vs 70.2 (SD 17.0)), 6 months (82.2 (SD 13.9) vs 74.9 (SD 16.7)) and 12 months (84.1 (SD 12.1) vs 75.6 (SD 17.0)). Cardiac rehabilitation and self-reported physical activity of $\geq 150 \mathrm{~min} /$ week were each positively associated with temporal improvements in HRQoL (coefficient: 2.12 (95\% Cl 0.68 to 3.55$)$ and 4.75 (95\% Cl 3.16 to 6.34$)$, respectively).

Conclusions Cardiac rehabilitation was independently associated with temporal improvements in HRQoL at up to 12 months following hospitalisation, with such changes further improved in patients who were physically active.

\section{INTRODUCTION}

Health-related quality of life (HRQoL) is an important outcome measure following acute myocardial infarction (AMI). ${ }^{1}$ Healthcare professionals have historically focused on objective measures of poor health, such as mortality and life expectancy, but often patients consider improvements to HRQoL equally important to length of life. ${ }^{2}$ The benefits of exercise post-AMI on mortality have been demonstrated since the early 1950 s, yet only recently has its potential to enhance HRQoL been recognised in this group. ${ }^{34}$

While cardiac rehabilitation has been associated with better HRQoL in patients following $\mathrm{AMI},{ }^{3}$ the majority of studies are limited by small sample sizes, cross-sectional designs, have limited generalisability or have not studied repeated (longitudinal) measures of HRQoL in populations. ${ }^{5-7}$ Moreover, the literature suggests that cardiac rehabilitation is an underused intervention with referral rates reported at around $50 \%$ and uptake frequently lower than this. ${ }^{89}$

The Evaluation of the Methods and Management of Acute Coronary Events (EMMACE)-3 is a national longitudinal prospective cohort study that collected data pertaining to the referral and uptake of cardiac rehabilitation in the National Health Service (NHS) of England. Among other patient-level items, it collected HRQoL scores, self-reported physical activity status, comorbidities, treatments and clinical outcomes for patients who were admitted to 48 hospitals between 1 November 2011 and 17 September 2013 with an acute coronary syndrome. ${ }^{10}$ This study aimed to investigate the association of cardiac rehabilitation and temporal changes in HRQoL estimated at multiple time points and to explore the impact of self-reported physical activity status on HRQoL trajectories.

\section{METHODS}

\section{Setting and design}

The study was based on data from 4570 patients who participated in EMMACE-3, a national longitudinal cohort study. ${ }^{10}$ Eligible patients included all adults aged 18 years and over hospitalised with AMI (ST-elevation myocardial infarction (STEMI) or non-ST-elevation myocardial infarction (NSTEMI)) who were admitted to 48 NHS hospitals in England between 1 November 2011 and 17 September 2013. Records for consenting patients were linked to the UK Myocardial Ischaemia National Audit Project (MINAP) to obtain data about their medical history, comorbidities, cardiac biomarkers, type of AMI (STEMI or NSTEMI), hospital treatments and discharge medications. ${ }^{11}$ The EMMACE-3 study protocol, of which this research is a subset, 
is available online (https://bmjopen.bmj.com/content/5/6/ e006256). ${ }^{10}$

\section{Assessment of HRQoL}

The primary outcome, self-reported HRQoL, was quantified using EuroQol 5-Dimension-3 Level Questionnaire (EQ-5D-3L), which has been validated in patients with $\mathrm{AMI}^{12}$ and consists of two component parts, a descriptive classification (EuroQol 5-Dimension (EQ-5D)) and a visual analogue scale (EuroQol 5-Visual Analogue Scale (EQ-VAS)). EQ-5D comprises five dimensions: mobility, self-care, usual activities, pain/discomfort and anxiety/depression, with each dimension subdivided into three levels: no problems, some problems, extreme problems, indicating a patient's perceived level of function. Each level carries a weighted score which is combined across the five dimensions to total a single index score, which is then standardised to the UK population. ${ }^{13}$ For the purposes of this study, we combined the levels 'some problems' and 'extreme problems' so that responses were binary. EQ-5D scores ranged from -0.5 to 1.0 , with negative scores indicating states 'worse than death', 0 indicating no quality of life or 'death', and 1 indicating full health. ${ }^{14} \mathrm{EQ}-\mathrm{VAS}$ is an analogue scale of $0-100$ in which participants are required to indicate their own perceived health, with 0 indicating 'worst imaginable health state' and 100 indicating 'best imaginable health state'. ${ }^{15} \mathrm{~A}$ difference in a score of 0.05 for EQ-5D and 7 for VAS is regarded as clinically important. ${ }^{16}$ For EMMACE-3, EQ-5D-3L data were collected at hospitalisation and at 30 days, and 6 and 12 months following discharge from the hospital.

\section{Statistical analyses}

Baseline characteristics for categorical data were described using frequencies and proportions. Normally distributed continuous data were described using means and SD, and non-normally distributed data were described using medians and IQRs. The differences in baseline characteristics between STEMI and NSTEMI were summarised using t-tests, $\chi^{2}$ tests, and Wilcoxon rank-sum tests appropriate to the data type and distribution.

Propensity score-based weighting coupled with a multilevel linear regression model (repeated measurements nested within patients and patients nested within hospitals) was applied to assess the relationship of attending cardiac rehabilitation on HRQoL. ${ }^{17}$ Briefly, the modelling involved a two-step approach: (1) a treatment assignment model estimating the propensity of attending cardiac rehabilitation at 30 days following discharge from the hospital was used to derive inverse-probability weights in order to balance any systematic differences in baseline characteristics between cardiac rehabilitation attendees and nonattendees (see online supplementary section 1 and 2) and (2) an outcome model assessing the impact of cardiac rehabilitation on HRQoL following AMI using a multilevel linear regression fitted to the weighted data at 30 days and 6 and 12 months.

Since the weighting was conducted using covariates observed at baseline, longitudinal multilevel regression models were additionally adjusted for the following patient-level factors in order to minimise potential residual confounding: baseline EQ-VAS, AMI phenotype (STEMI vs NSTEMI), sex, age, Body Mass Index (BMI), Index of Multiple Deprivation (IMD) score, smoking status (never vs current or ex-smoker), family history of coronary heart disease (CHD), previous angina, history of diabetes mellitus, hypertension, heart failure, peripheral vascular disease, cerebrovascular disease, chronic renal failure, chronic obstructive pulmonary disease (COPD) or asthma, hypercholesterolaemia, previous percutaneous coronary intervention (PCI), previous coronary artery bypass graft (CABG) surgery, previous AMI, PCI during AMI hospitalisation, CABG surgery during AMI hospitalisation, reinfarction during index AMI hospitalisation, medications prescribed at discharge from the hospital (including aspirin, beta-blockers, statins and ACE inhibitors) and self-reported physical activity status. Missing data were imputed using multiple imputation by chained equations (10 datasets from 20 iterations), and a default imputation strategy based on clinical expert opinion was implemented for selected treatment variables (online supplementary section 2). Pooled estimates and accompanying 95\% CIs for each model were generated according to Rubin's rules. ${ }^{18}$ Analysis were performed using STATA MP64 V.14 (StataCorp,www.stata.com), and $\mathrm{p}$ values of $<0.05$ were considered statistically significant.

\section{Patient involvement}

While no patients were involved in setting the research question or the study design, the work is coauthored by a patient representative $(\mathrm{GO})$ who helped with the interpretation of the findings and provided a critical review of the manuscript text.

\section{RESULTS}

\section{Study sample}

From 5557 hospitalisations of consented patients across 48 hospitals, $181(3.3 \%)$ patients withdrew from the study; we excluded $510(9.2 \%)$ due to failed data linkage to MINAP and 296 (5.3\%) who did not have a discharge diagnosis of AMI, leaving an analytical cohort of 4570 (online supplementary eFigure 1). For the weighted multilevel linear regression model, we analysed an effective sample size of 3438 because a further 1132 patients were excluded due to missing exposure/outcome data. Questionnaire response rates were 96.4\% (4403/4570), 74.3\% (3395/4570), 65.1\% (2973/4570) and 61.9\% (2828/4570) at hospitalisation, 30 days, and 6 and 12 months, respectively. Sixteen patients $(0.3 \%)$ died in the hospital. Missing data levels were $<7 \%$ for baseline patient demographic characteristics, except for IMD (5.8\%), BMI (39.3\%), family history of CHD $(13.1 \%)$ and ethnicity $(22.0 \%)$ (table 1$)$. The mean age for the analytical cohort was 63.6 (SD 11.9) years; $25.2 \%$ were women; $76.3 \%$ were Caucasian; the median BMI was 27.8 (25.1-31.3) $\mathrm{kg} / \mathrm{m}^{2}$; the median IMD score was 18.5 (IQR 11.0-31.8); and $65.9 \%$ were current or ex-smokers. Comorbidity was common, including hypertension $(42.7 \%)$, angina $(19.6 \%)$, diabetes mellitus (15.9\%) and COPD or asthma (12.1\%) (table 1).

\section{HRQoL trajectories}

For EQ-5D dimensions, 66.6\% (2893/4344) of participants reported $\geq 1$ problem at hospitalisation, which increased to $70.8 \%(2356 / 3326)$ at 30 days, then decreased to $58.4 \%$ $(1712 / 2930)$ at 6 months, and $56.9 \%(1584 / 2783)$ at 12 months. Participants reported having the greatest burden of problems (of any level) at hospitalisation in usual activities (45.8\%), followed by mobility $(35.5 \%)$, pain $(32.9 \%)$, anxiety $(30.2 \%)$ and selfcare (13.8\%) (figure 1 and online supplementary eTable 4). Each domain improved at each time point over the 12-month period except for self-care, which showed a modest increase from $7.7 \%$ to $8.2 \%$ at 6 and 12 months, respectively. Compared with the UK averages, participants reported a greater proportion of problems in usual activities, self-care and mobility at all time points. Anxiety was reported at a greater proportion at hospitalisation and 30 days; however, this then improved to below the UK average at 6 and 12 months. At hospitalisation, 
Table 1 Patient baseline characteristics stratified by type of acute myocardial infarction (STEMI vs NSTEMI)

\begin{tabular}{|c|c|c|c|c|c|}
\hline & STEMI, $\mathrm{n}=1856$ & NSTEMI, $\mathrm{n}=2714$ & $P$ value & All AMI, $n=4570$ & Missing, $\mathrm{n}(\%)$ \\
\hline \multicolumn{6}{|l|}{ Variables } \\
\hline Age (years), mean (SD) & $60.9(11.5)$ & $65.5(11.9)$ & $<0.001$ & $63.6(11.9)$ & $17(0.4)$ \\
\hline Women, $\mathrm{n}(\%)$ & $432(23.3)$ & $720(26.6)$ & 0.012 & $1152(25.2)$ & $15(0.3)$ \\
\hline IMD score, median (IQR) & $19.1(11.0-34.2)$ & $18.1(11.0-30.4)$ & 0.010 & $18.5(11.0-31.8)$ & $264(5.8)$ \\
\hline BMI, median (IQR), kg/ m² & $27.3(24.8-30.9)$ & $28.2(25.3-31.7)$ & $<0.001$ & $27.8(25.1-31.3)$ & $1794(39.3)$ \\
\hline Ex/current smoking status, $n$ (\%) & $1251(67.4)$ & $1761(64.9)$ & $<0.001$ & $3012(65.9)$ & $143(3.1)$ \\
\hline Caucasian, n (\%) & $1415(76.2)$ & $2071(76.3)$ & 0.218 & $3486(76.3)$ & $1004(22.0)$ \\
\hline Family history of CHD, $\mathrm{n}(\%)$ & $696(37.5)$ & $998(36.8)$ & 0.295 & $1694(37.1)$ & 599 (13.1) \\
\hline \multicolumn{6}{|l|}{ Comorbidities } \\
\hline Previous $\mathrm{PCl}, \mathrm{n}(\%)$ & $68(3.7)$ & $245(9.0)$ & $<0.001$ & $313(6.9)$ & $170(3.7)$ \\
\hline Previous CABG surgery, $\mathrm{n}(\%)$ & $39(2.1)$ & $251(9.3)$ & $<0.001$ & $290(6.4)$ & $168(3.7)$ \\
\hline Previous AMI, n (\%) & $127(6.8)$ & $506(18.6)$ & $<0.001$ & $633(13.9)$ & $169(3.7)$ \\
\hline Previous angina, $\mathrm{n}(\%)$ & $162(8.7)$ & $734(27.0)$ & $<0.001$ & $896(19.6)$ & $169(3.7)$ \\
\hline Chronic renal failure, $\mathrm{n}(\%)$ & $17(0.9)$ & $106(3.9)$ & $<0.001$ & $123(2.69 \%)$ & $180(3.9)$ \\
\hline Hypertension, n (\%) & $644(34.7)$ & $1306(48.1)$ & $<0.001$ & $1950(42.7)$ & $178(3.9)$ \\
\hline Chronic heart failure, $\mathrm{n}(\%)$ & $6(0.3)$ & $72(2.7)$ & $<0.001$ & $78(1.71)$ & $177(3.9)$ \\
\hline Hypercholesterolaemia, n (\%) & $469(25.3)$ & $927(34.2)$ & $<0.001$ & $1396(30.6)$ & $216(4.7)$ \\
\hline Peripheral vascular disease, $\mathrm{n}(\%)$ & $43(2.3)$ & $109(4.0)$ & 0.002 & $152(3.3)$ & $302(6.6)$ \\
\hline Asthma/COPD, n (\%) & $184(9.9)$ & $370(13.6)$ & 0.001 & $554(12.1)$ & $188(4.1)$ \\
\hline Cerebrovascular disease, $\mathrm{n}(\%)$ & $52(2.8)$ & $134(4.9)$ & 0.001 & $186(4.1)$ & $180(3.9)$ \\
\hline Diabetes mellitus, n (\%) & $202(10.9)$ & $524(19.3)$ & $<0.001$ & $726(15.9)$ & $93(2.0)$ \\
\hline \multicolumn{6}{|l|}{ Treatments during hospitalisation } \\
\hline $\mathrm{PCl}, \mathrm{n}(\%)$ & $654(35.2)$ & $1169(43.1)$ & $<0.001$ & $1823(39.9)$ & $735(16.1)$ \\
\hline CABG surgery, $n(\%)$ & $36(1.9)$ & $296(10.9)$ & $<0.001$ & $332(7.3)$ & $735(16.1)$ \\
\hline \multicolumn{6}{|l|}{ Discharge medications } \\
\hline Aspirin, $\mathrm{n}(\%)$ & $1720(92.7)$ & $2228(82.1)$ & $<0.001$ & $3948(86.4)$ & $142(3.1)$ \\
\hline Beta blocker, n (\%) & $1650(88.9)$ & $2031(74.8)$ & $<0.001$ & $3681(80.6)$ & $144(3.2)$ \\
\hline Statins, $n(\%)$ & $1714(92.4)$ & $2245(82.7)$ & $<0.001$ & 3959 (86.6) & $141(3.1)$ \\
\hline ACEi, $n(\%)$ & $1658(89.3)$ & $2061(75.9)$ & $<0.001$ & $3719(81.4)$ & $161(3.5)$ \\
\hline \multicolumn{6}{|l|}{ Cardiac rehabilitation } \\
\hline $\begin{array}{l}\text { Cardiac rehabilitation offered at } \\
\text { baseline, } \mathrm{n}(\%)\end{array}$ & 1734 (93.4) & $2467(90.9)$ & $<0.001$ & $4201(91.9)$ & $264(5.8)$ \\
\hline \multicolumn{6}{|l|}{ Adverse cardiac events } \\
\hline Deaths in hospital n (\%) & $2(0.1)$ & $14(0.5)$ & 0.022 & $16(0.3)$ & $61(1.3)$ \\
\hline Reinfarction in hospital n (\%) & $16(0.9)$ & $14(0.5)$ & 0.153 & $30(0.7)$ & $151(3.3)$ \\
\hline \multicolumn{6}{|l|}{ HRQoL } \\
\hline Baseline EQ-VAS, mean (SD) & $65.0(19.8)$ & $63.8(20.0)$ & 0.051 & $64.3(19.9)$ & $193(4.2)$ \\
\hline
\end{tabular}

ACEi, ACE inhibitor; AMI, acute myocardial infarction; BMI, Body Mass Index; CABG, coronary artery bypass graft; CHD, coronary heart disease; COPD, chronic obstructive pulmonary disease; HRQoL, health-related quality of life; IMD, Index of Multiple Deprivation; NSTEMI, non-ST-elevation myocardial infarction; PCI, percutaneous coronary intervention; STEMI, ST-elevation myocardial infarction.

pain was reported at a similar proportion to the UK average but improved at each subsequent follow-up point. Patients with NSTEMI reported an increased frequency of $\geq 1$ problem at all time points, at hospitalisation $(65.5 \%$ vs $60.1 \%), 30$ days (53.4\% vs $48.9 \%), 6$ months (39.9\% vs 33.9) and 12 months $(37.7 \%$ vs $30.2 \%)$. This pattern of improvement from hospitalisation to 12 months was also reflected in the index scores of all patients with AMI (EQ-5D-3L: 0.74 (SD 0.28) vs 0.79 (SD 0.26); EQ-VAS: 64.3 (SD 19.9) vs 74.4 (SD 18.5)) (table 2). Patients with NSTEMI reported worse HRQoL than those with STEMI at each time point except at baseline, such that the mean EQ-5D-3L scores and EQ-VAS scores were 0.74 vs 0.78 and 69.6 vs 71.2 at 30 days, 0.78 vs 0.81 and 72.9 vs 75.3 at 6 months, and 0.78 vs 0.82 and 73.1 vs 76.5 at 12 months, respectively, for NSTEMI compared with STEMI (online supplementary eTable 5). Furthermore, those who attended cardiac rehabilitation and reported physical activity of $\geq 150 \mathrm{~min} /$ week had greater temporal improvements in HRQoL scores compared with those who attended and reported physical activity of $\leq 150 \mathrm{~min} /$ week: 30 days (mean EQ-VAS: 79.3 (SD 14.6) vs 70.2 (SD 17.0)), 6 months (EQ-VAS: 82.2 (SD 13.9) vs 74.9 (SD 16.7)) and 12 months (EQ-VAS: 84.1 (SD 12.1) vs 75.6 (SD 17.0)) (figure 2 and online supplementary eTable 6).

\section{Association of cardiac rehabilitation with HRQoL trajectories}

Participants who attended cardiac rehabilitation had higher HRQoL scores compared with those who did not attend. Moreover, attendees showed a greater temporal improvement at each follow-up point: 30 days (mean EQ-VAS: 71.0 (SD 16.8) vs 68.6 (SD 19.8)), 6 months (EQ-VAS: 76.0 (SD 16.4) vs 70.2 (SD 19.0)) and 12 months (EQ-VAS: 76.9 (SD 16.8) vs 70.4 (SD 20.4)). The propensity weighted multilevel model demonstrated temporal improvements in HRQoL following AMI over the 12-month follow-up period (EQ-VAS score coefficient at 6 months: 3.18 (95\% CI 2.22 to 4.14 ) and at 12 months: 3.81 


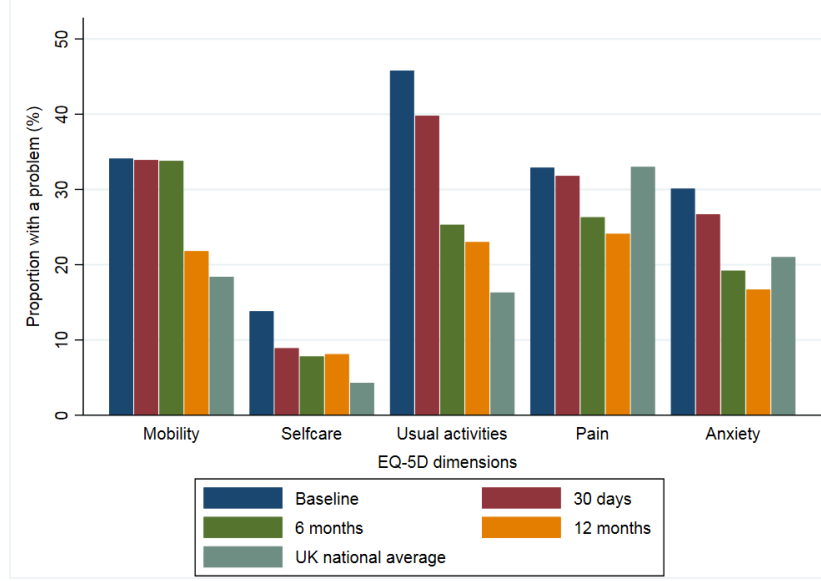

Figure 1 Proportion of patients with acute myocardial infarction who reported $\geq 1$ problem across all of the EuroQol 5-Dimension domains at baseline, 30 days, and 6 and 12 months of follow-up. UK national average included.

(95\% CI 2.72 to 4.90 ) compared with 30 days). Cardiac rehabilitation and self-reported physical activity of $\geq 150 \mathrm{~min} /$ week were also positively associated with HRQoL (2.12 (95\% CI 0.68 to 3.55 ) and 4.75 (95\% CI 3.16 to 6.34), respectively) (figure 3 ). After standardisation for case mix, there was minimal variance between hospitals. However, there was wide variation between patients (48.7\%) and within patients over time (51.3\%) (online supplementary eTable 7).

\section{DISCUSSION}

In this national longitudinal study of 4570 patients hospitalised with AMI, we found that attendance at cardiac rehabilitation was associated with a temporal improvement in HRQoL at up to 12 months following hospital discharge. Moreover, for patients who also participated in activities of $\geq 150 \mathrm{~min} / \mathrm{week}$, the magnitude of the positive association between cardiac rehabilitation and improvements in HRQoL was larger.

It has previously been suggested that the minimal clinically important difference for HRQoL as measured by EQ-VAS is 7 points. ${ }^{16}$ We found that at 12 months, the absolute difference in EQ-VAS was 14.9 points when comparing patients who both attended cardiac rehabilitation and undertook additional physical activity with those who did neither (84.1 vs 69.2). Moreover, the absolute difference in EQ-VAS was 6.5 when comparing

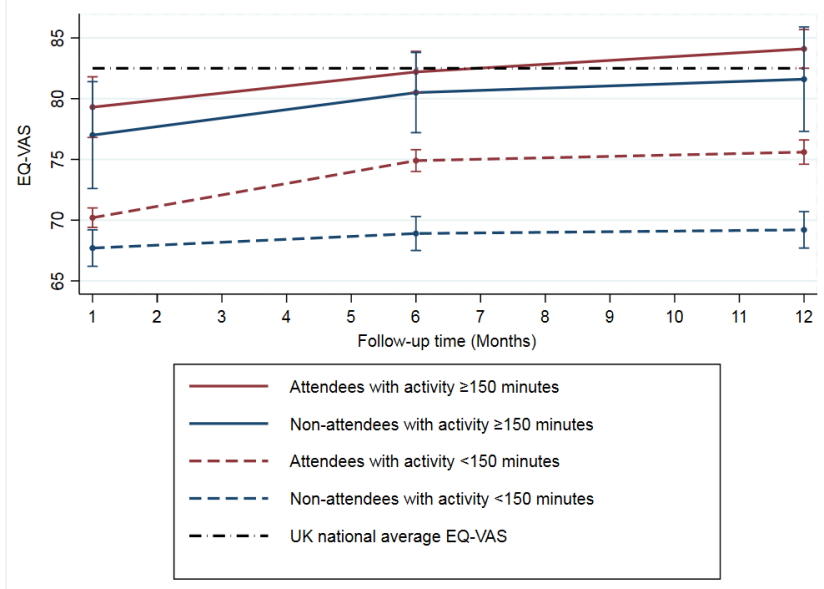

Figure 2 Health-related quality of life trajectories of patients with acute myocardial infarction according to their attendance at cardiac rehabilitation and/or self-reported physical activity of $\geq 150 \mathrm{~min} /$ week.

attendees to non-attendees of cardiac rehabilitation alone (76.9 vs 70.4). The propensity score wei

ghted modelling, on the other hand, found that attending cardiac rehabilitation and physical activity of $\geq 150 \mathrm{~min} /$ week at 30 days had a positive impact on changes in HRQoL (EQ-VAS score coefficient of 2.12 (95\% CI 0.68 to 3.55 ) and 4.75 (95\% CI 3.16 to 6.34$)$ ). This relates to the average relative increase over the follow-up period. Although the coefficients are not equal or greater than 7 , we considered the positive impact clinically relevant.

Our results build on the existing literature. A systematic review of randomised controlled trials reported improvements in HRQoL measures in 14/20 studies for patients with AMI, previous coronary revascularisation or established CHD who participated in an exercise-based cardiac rehabilitation programme compared with those who received standard care. ${ }^{4}$ However, they were unable to undertake a meta-analysis due to heterogeneity in outcome measures and reporting methods. Many previous studies have also been limited by small sample sizes and non-randomisation of treatment arms or have been predominantly not of UK/European cohorts. ${ }^{19-22}$

Notably, the rehabilitation after myocardial infarction (randomised controlled) trial (RAMIT) found no significant effect of cardiac rehabilitation on mortality or HRQoL. ${ }^{23}$ Much has been debated regarding these findings particularly as the

Table 2 Mean EQ-5D-3L and EQ-VAS scores at baseline, 30 days, and 6 and 12 months of patients attending cardiac rehabilitation versus those who did not attend

\begin{tabular}{|c|c|c|c|c|c|}
\hline Variable & Attended cardiac rehabilitation & Did not attend cardiac rehabilitation & $P$ value & All AMI, $n=4570$ & Missing, $\mathrm{n}(\%)$ \\
\hline \multicolumn{6}{|c|}{ EQ-5D Index score, mean (SD) } \\
\hline Hospitalisation (SD) & $0.766(0.264), n=1681$ & $0.754(0.277), n=874$ & 0.302 & $0.744(0.280)$ & $226(5.0)$ \\
\hline 30 days, mean (SD) & $0.773(0.232), n=2259$ & $0.728(0.278), n=874$ & $<0.001$ & $0.757(0.250)$ & $1244(27.2)$ \\
\hline 6 months, mean (SD) & $0.821(0.236), n=1862$ & $0.737(0.290), n=885$ & $<0.001$ & $0.790(0.261)$ & $1640(35.9)$ \\
\hline 12 months, mean (SD) & $0.832(0.225), n=1725$ & $0.739(0.294), n=887$ & $<0.001$ & $0.794(0.260)$ & $1787(39.1)$ \\
\hline \multicolumn{6}{|c|}{ EQ-5D VAS score, mean (SD) } \\
\hline Hospitalisation (SD) & $65.4(19.6) n=1,694$ & $64.5(20.0), n=880$ & 0.312 & $64.3(19.9)$ & $193(4.2)$ \\
\hline 30 days, mean (SD) & $71.0(16.8), n=2251$ & $68.6(19.7), n=865$ & $<0.001$ & $70.2(17.8)$ & $1269(27.8)$ \\
\hline 6 months, mean (SD) & $76.0(16.4), n=1841$ & $70.2(19.0), n=867$ & $<0.001$ & $73.9(17.7)$ & $1691(37.0)$ \\
\hline 12 months, mean (SD) & $76.9(16.8), n=1691$ & $70.4(20.3), n=884$ & $<0.001$ & $74.4(18.5)$ & $1840(40.3)$ \\
\hline
\end{tabular}

EQ-5D-3L, EuroQol 5-Dimension-3 Level Questionnaire; EQ-VAS, EuroQol Visual Analogue Scale. 

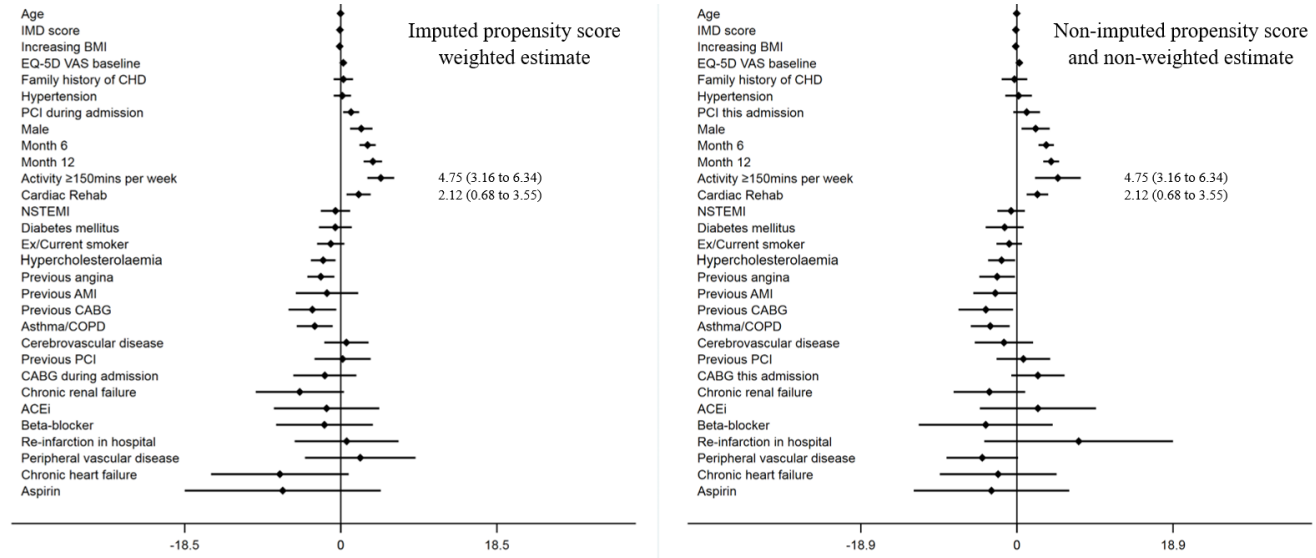

Figure 3 Results of the imputed propensity score weighted multi-level modelling of the association of cardiac rehabilitation and change in EQ-VAS following AMI (regression coefficients, 95\% confidence intervals). ACEi, ACE inhibitor; AMI, acute myocardial infarction; BMI, Body Mass Index; CABG, coronary artery bypass graft; CHD, coronary heart disease; COPD, chronic obstructive pulmonary disease; EQ-5D VAS, EuroQol 5-dimension Visual Analogue Scale; IMD, Index of Multiple Deprivation; PCI, percutaneous coronary intervention; NSTEMI, non-ST-elevation myocardial infarction.

majority of the evidence for cardiac rehabilitation is from studies conducted prior to modern advancements in pharmacotherapeutic and invasive coronary strategies; it has been suggested that cardiac rehabilitation may no longer possess significant benefits for patients. ${ }^{24}$ However, there were a number of issues which may have contributed to the results of RAMIT, including lack of sufficient study power and a potential loss of clinical equipoise. The UK National Audit of Cardiac Rehabilitation reports that only $<30 \%$ of patients offered cardiac rehabilitation declined attending, whereas in RAMIT, there was a 50\% risk that participants would miss out on cardiac rehabilitation and therefore may not have participated. ${ }^{25} 26$

Observational longitudinal population-based studies such as ours negate some of the above issues by allowing the nesting of time periods, which enables investigation of trajectories of HRQoL and potentially within a much larger cohort of patients over time. Our data suggest that cardiac rehabilitation has an important role to play in HRQoL recovery after AMI, and encouragement of sustained physical activity beyond the initial period of cardiac rehabilitation may lead to even greater benefits in HRQoL over time.

While there are many strengths to this work, including its nationwide and longitudinal design and the efficiency of data enhancement through linkage to national clinical registries, we acknowledge the inherent weakness in the observational design. Foremost is that we do not propose a causal relationship, which can only be tested in randomised studies. Nevertheless, we undertook a weighted propensity score analysis which will, in part, have mitigated some of the confounding by indication. This analysis supports the relationship between cardiac rehabilitation and temporal improvements in HRQoL. Although we used a generic HRQoL indicator, this has previously been validated in patients post-AMI, and the domains are typically expected to be affected by AMI. ${ }^{12}$ There was a loss to follow-up of nearly $40 \%$ by 12 months, which is not unexpected when conducting cohort studies. This may, however, have resulted in a selection bias should those lost had worse HRQoL. Notwithstanding this, the multilevel model technique enabled inclusion of all patients in the analysis, even if they were not assessed at all follow-up points. There is also potential for recall bias or misclassification in respect to self-reported physical activity, given that patients had to report how often they were physically active for more than $15 \mathrm{~min}$ in a week and whether this was of light, moderate or strenuous in intensity. As with all observational studies, there is a possibility that HRQoL after AMI is affected by unmeasured confounders, which has not been accounted for in the analysis, including coronary anatomy, other comorbidities, variation between cardiac rehabilitation programmes, acuity of presentation and also acuity of intervention.

\section{CONCLUSIONS}

This national cohort study of 4570 patients demonstrated that cardiac rehabilitation was associated with improved HRQoL. Furthermore, it suggests that these benefits were even greater for those who participated in $\geq 150$ min of physical activity per week. Therefore, encouraging patients to participate in cardiac rehabilitation and to pursue sustained physical activity may be important for recovery post-AMI. Further studies are required

\section{Key messages}

What is already known on this subject?

- Cardiac rehabilitation is associated with better health-related quality of life (HRQoL) following acute myocardial infarction (AMI).

- However, there is a paucity of information about the impact of cardiac rehabilitation on temporal changes in $\mathrm{HRQ}$ oL in the modern pharmacotherapeutic and invasive coronary era.

- To date, there are no published nationwide longitudinal studies of repeated measures of perceived HRQoL following AMI.

\section{What might this study add?}

- This national longitudinal cohort study across 48 hospitals of 4570 patients with AMI found that attendance at a cardiac rehabilitation programme was independently associated with temporal improvements in HRQoL at up to 12 months following hospitalisation, with such changes further improved in patients who were physically active.

\section{How might this impact on clinical practice?}

- Ensuring the provision of cardiac rehabilitation services that enable all patients with AMI to attend may be an important determinant of the recovery of HRQoL. 
to determine if the dose of exercise training and or heightened levels of physical activity status alongside cardiac rehabilitation may result in even greater benefits for patients.

\section{Author affiliations}

${ }^{1}$ Leeds Institute for Data Analytics, Leeds Institute of Cardiovascular and Metabolic Medicine, Leeds, UK

${ }^{2}$ Department of Cardiology, Leeds Teaching Hospitals NHS Trust, Leeds, UK

${ }^{3}$ Patient Collaborator, Lancashire, UK

${ }^{4}$ Health Sciences, University of York, York, UK

Twitter Ben Hurdus @benhurdus, Tatendashe Bernadette Dondo @ bernadetteTDond and Chris P Gale @cpgale3

Acknowledgements We gratefully acknowledge the contributions from all hospitals, healthcare professionals and patients who participated in the Evaluation of the Methods and Management of Acute Coronary Events study. We also thank Richard Gillott (IT support), Claire Forrest (cardiology research coordinator) and Vera Hall (finance support).

Contributors $\mathrm{BH}$ analysed the data and drafted the manuscript. CPG contributed to the design of the study, provided clinical expert advice in interpretation of the results and was involved in manuscript writing. TM was involved in the design of the study, statistical advice, data analysis and writing of the manuscript. TBD was involved in statistical advice, data analysis and writing of the manuscript. ASH contributed to the design of the study and manuscript writing. SA, MH and PD were involved in interpretation of data and manuscript writing. $\mathrm{GO}$ was involved as a patient advisor in the interpretation of the research and the writing of the manuscript. CPG and BH are the guarantors for this study. All authors made critical revisions and provided intellectual content to the manuscript, approved the final version to be published and agreed to be accountable for all aspects of the work.

Funding This research was funded by the National Institute for Health Research (NIHR/CS/009/004), and British Heart foundation (PG/19/54/34511).

\section{Competing interests None declared.}

Patient and public involvement Patients and/or the public were involved in the design, conduct, reporting or dissemination plans of this research. Refer to the Methods section for further details.

Patient consent for publication Not required.

Ethics approval EMMACE-3 has been given a favourable ethical opinion by the Leeds (Central) Research Ethics committee (REC reference: 10/H1313/74), is registered on ClinicalTrials.gov (NCT01808027) and has been adopted onto the National Institute for Health Research Comprehensive Research Network portfolio (9102).

Provenance and peer review Not commissioned; externally peer reviewed.

Data availability statement Data are available upon reasonable request. All data relevant to the study are included in the article or uploaded as supplementary information. The data that support the findings of this study is available in the supplement and also available from the corresponding author, $\mathrm{BH}$, upon reasonable request.

Open access This is an open access article distributed in accordance with the Creative Commons Attribution 4.0 Unported (CC BY 4.0) license, which permits others to copy, redistribute, remix, transform and build upon this work for any purpose, provided the original work is properly cited, a link to the licence is given, and indication of whether changes were made. See: https://creativecommons.org/ licenses/by/4.0/.

\section{ORCID iDs}

Ben Hurdus http://orcid.org/0000-0001-8149-3449

Theresa Munyombwe http://orcid.org/0000-0002-1307-6691

Tatendashe Bernadette Dondo http://orcid.org/0000-0002-8337-8425

\section{REFERENCES}

1 Munyombwe T, Hall M, Dondo TB, et al. Quality of life trajectories in survivors of acute myocardial infarction: a national longitudinal study. Heart 2020;106:33-9.

2 Thompson DR, Yu C-M. Quality of life in patients with coronary heart disease-l: assessment tools. Health Qual Life Outcomes 2003;1:42.

3 Antonakoudis H, Kifnidis K, Andreadis A, et al. Cardiac rehabilitation effects on quality of life in patients after acute myocardial infarction. Hippokratia 2006;10:176-81.

4 Anderson L, Oldridge N, Thompson DR, et al. Exercise-Based Cardiac Rehabilitation for Coronary Heart Disease. J Am Coll Cardiol 2016;67:1-12.

5 Mollon L, Bhattacharjee S. Health related quality of life among myocardial infarction survivors in the United States: a propensity score matched analysis. Health Qual Life Outcomes 2017:15:235.

6 Staniūtè M, Brožaitiene J. Changes in health-related quality of life among patients with coronary artery disease: a 2-year follow-up. Medicina 2010:46:843.

7 Bahall M, Khan K. Quality of life of patients with first-time AMI: a descriptive study. Health Qual Life Outcomes 2018:16:32.

8 Brown T, Hernandez A, Bittner V, et al. On behalf of the American heart association get with the guidelines Investigators. predictors of cardiac rehabilitation referral in coronary artery disease patients. J Am Coll Cardiol 2009;54:515-21.

9 Aragam KG, Moscucci M, Smith DE, et al. Trends and disparities in referral to cardiac rehabilitation after percutaneous coronary intervention. Am Heart $J$ 2011;161:544-51.

10 Alabas OA, West RM, Gillott RG, et al. on behalf of the EMMACE-3 Investigators, et al. Evaluation of the Methods and Management of Acute Coronary Events (EMMACE)-3: protocol for a longitudinal study. BMJ Open 2015:5:e006256.

11 Herrett E, Smeeth L, Walker L, et al. The myocardial ischaemia national audit project (MINAP). Heart 2010:96:1264-7.

12 Nowels D, McGloin J, Westfall JM, et al. Validation of the EQ-5D quality of life instrument in patients after myocardial infarction. Qual Life Res 2005;14:95-105 https://doi.org/

13 EuroQol Research Foundation. EQ-5D-3L user guide, 2018. Available: https://eurogol. org/publications/user-guides

14 Mulhern B, Feng Y, Shah K, et al. Comparing the UK EQ-5D-3L and English EQ-5D-5L value sets. Pharmacoeconomics 2018:36:699-713.

15 Feng Y, Parkin D, Devlin NJ. Assessing the performance of the EQ-VAS in the NHS PROMs programme. Qual Life Res 2014:23:977-89.

16 Nolan CM, Longworth L, Lord J, et al. The EQ-5D-5L health status questionnaire in COPD: validity, responsiveness and minimum important difference. Thorax 2016;71:493-500

17 Goldstein H, Browne W, Rasbash J. Multilevel modelling of medical data. Stat Med 2002:21:3291-315.

18 Rubin DB. Multiple imputation for nonresponse in surveys. 81. John Wiley \& Sons, 2004

19 Wang W, Chair SY, Thompson DR, et al. Effects of home-based rehabilitation on health-related quality of life and psychological status in Chinese patients recovering from acute myocardial infarction. Heart Lung 2012;41:15-25.

20 Reid RD, Morrin LI, Beaton LJ, et al. Randomized trial of an Internet-based computertailored expert system for physical activity in patients with heart disease. Eur J Prev Cardiol 2012:19:1357-64.

21 Mutwalli HA, Fallows SJ, Arnous AA, et al. Randomized controlled evaluation shows the effectiveness of a home-based cardiac rehabilitation program. Saudi Med J 2012;33:152-9.

22 Maddison R, Pfaeffli L, Whittaker R, et al. A mobile phone intervention increases physical activity in people with cardiovascular disease: results from the heart randomized controlled trial. Eur J Prev Cardiol 2015;22:701-9.

23 West RR, Jones DA, Henderson AH. Rehabilitation after myocardial infarction trial (RAMIT): multi-centre randomised controlled trial of comprehensive cardiac rehabilitation in patients following acute myocardial infarction. Heart 2012:98:637-44.

24 Rauch B, Davos CH, Doherty P, et al. The prognostic effect of cardiac rehabilitation in the era of acute revascularisation and statin therapy: A systematic review and metaanalysis of randomized and non-randomized studies - The Cardiac Rehabilitation Outcome Study (CROS). Eur J Prev Cardiol 2016;23:1914-39.

25 Doherty P, Lewin R. The RAMIT trial, a pragmatic RCT of cardiac rehabilitation versus usual care: what does it tell us? Heart 2012:98:605-6.

26 Doherty P, Harrison AS. The National audit of cardiac rehabilitation: quality and outcomes report. London: British Heart Foundation, 2018. 